\title{
When Matching up Features Messes up Decisions: The Role of Feature Matching in Successive Choices
}

\author{
Sara D. Hodges \\ University of Oregon
}

\begin{abstract}
Two studies examined feature matching in successive judgments. College students in both studies read about 2 apartments with shared positive and unique negative features, and 1 less globally positive apartment with all unique features. In the feature-cancellation condition, participants first saw the shared-features apartments together and then saw the all-unique apartment. In the no-cancellation condition, participants first saw 1 shared-features apartment and the all-unique apartment together and then saw the other shared-features apartment. Shared-features apartments were rated higher when they were not seen together. The all-unique apartment was rated higher when it was seen last, when the first 2 apartments' shared features canceled out. Study 2 replicated Study 1 and also demonstrated that 2 additional variables failed to moderate the effect. Memory measures suggest that shared features are paired up as an organizational strategy.
\end{abstract}

During the 1992 U.S. Presidential election, the country let out a collective whine when presented with the Democratic and Republican candidates. Instead of just expressing the usual apathy about the election, voters seemed actively critical of both of the front-runners. George Bush was seen as being out of touch with middle- and lower-class Americans, too concerned with remote international affairs, and responsible for the country's domestic and economic problems. Bill Clinton was disliked for a different reason: allegations of sins in the past (extramarital affairs, drug use, and draft dodging), which he avoided facing directly in his inimitable "Slick Willy" style. The choice between the two candidates was painted as a decision between the lesser of two evils, and the many overlapping positive qualities of the two front-runners seemed largely ignored. Both were tall, White, male, Ivy League graduates with a great deal of political experience, and both were quite moderate and mainstream, but voters seemed to pay little attention to these similar positive qualifications and instead largely focused on the divisive negative features of Bush and Clinton.

Enter third-party candidate Ross Perot, who, despite a dearth of experience in politics, was embraced by Americans as no other third-party candidate had been in recent elections. Clearly, Perot's campaign had a number of advantages, many of them related to his great wealth, but one less obvious help may have been Perot's late entry into a race that already contained two

This article is based on my doctoral dissertation, submitted to the University of Virginia. I warmly acknowledge my dissertation committee, headed by Timothy Wilson and composed of Susan Brodt, Bella DePaulo, Charlie Holt, and Dan Wegner. I also extend thanks to Michelle Kongkasuwan and Jennifer Lacey for their help in collecting the data and to Jim Sherman and David Houston for feedback on this article.

Correspondence concerning this article should be addressed to Sara D. Hodges, Department of Psychology, 1227 University of Oregon, Eugene, Oregon 97403-1227. Electronic mail may be sent via the Internet to sdhodges@darkwing.uoregon.edu. candidates who overlapped on positive features but had unique negative features.

It is difficult to judge the validity of a post hoc explanation of the election, but the 1992 Presidential election serves as a compelling real-life example of a phenomenon addressed by the present studies. When given a choice between two options that have some shared features, and some features that are unique to only one of the options, people have a tendency to "match up" the features, cancel out the shared features, and base their decision solely on the unique features (Houston \& Sherman, 1995; Houston, Sherman, \& Baker, 1989, 1991). In particular, people pay special attention to the unique features of the subject of comparison (often, the most recent option to be seen ), which is the option compared with some other standard or criterion, known as the object of comparison. The present studies push feature matching further, specifically examining whether people continue to use only the unique features of earlier options when comparing them to options that are later added to the choice field.

Feature matching, or the cognitive comparison strategy of matching up the features shared by two options, was originally identified by Tversky (1977) as a strategy for judging similarity but has also been found to play a part in preference judgments (Houston \& Sherman, 1995; Houston et al., 1989, 1991; Kardes \& Sanbonmatsu, 1993). With a choice between two options, this strategy can be efficient and effective-people concern themselves only with the unique dimensions on which the two options differ, not with overlapping features that fail to distinguish between the two choices.

However, the strategy is not without side effects. Houston et al. (1991) showed that removing the shared features from a decision context changes the nature of the decision. When shared features are not used in the preference process, a choice between two options that have shared positive features and unique negatives is like a choice between two negatives-an avoidance-avoidance conflict. On the other hand, a choice between two options that share negative aspects but have unique 
positives is like an approach-approach conflict. We should expect people to behave differently if they have transformed the decision from one that contains some pros and some cons into one that is a choice between exclusively negative options, especially given that other areas of social psychology have found negative features to be more potent than positive features (Kahneman \& Tversky, 1984; Skowronski \& Carlston, 1989). However, the notable twist to Houston et al.'s (1991) study is that it was the participants themselves who changed the nature of the choice by feature matching and turned initially equivalent options with equal numbers of negative and positive features into a choice between the lesser of two evils, or the greater of two boons. Not surprisingly, Houston et al. (1991) found that this framing of options affects not only how we see our choice but also our postdecisional feelings-it's difficult to be happy with your choice if you saw yourself losing either way, just as it's easy to be happy if you considered both options good. Furthermore, Houston and Sherman (1995) found that not only did people have less happy postdecisional feelings, but they also gave lower postchoice evaluations to items that had been seen in shared-positive-unique-negative pairs than to the same items when they had been seen in shared-negative-unique-positive pairs.

Does the context created by feature matching persist beyond the original decision between the first two options? If so, then a later option subsequently added to the choice field may be seen in a different light. The role of feature matching in successive decisions with more than two options was foreshadowed by earlier decision researchers who theorized that expanding the number of options in a choice set would reduce the probability that one of the options would be chosen. Thus, the probability that an option would be chosen was greater in smaller choice sets, but only to the extent that the options did not overlap (see Luce, 1959; Restle, 1961; Rumelhart \& Greeno, 1971). In terms of adding more options to the choice set, generally " the addition of an alternative to an offered set 'hurts' alternatives that are similar to the added alternative more than those that are dissimilar to it"' (Tversky, 1972, p. 283).

The present studies investigated a possible negative side effect of feature matching and canceling when a third option is belatedly added to a choice field already containing options that have some similarities. If people have been concentrating mostly on the unique features of earlier options, they may continue to use only these unique features - they may fail to go back and retrieve any features shared by earlier options when comparing the earlier options with a later option. In other words, for some options (the earlier ones), only a subset of features will be compared with a full set of features for other options (the later ones). It is as if the features shared by the earlier options have been removed from the field, or canceled out, leaving only unique features to be compared with later options.

Which features get matched up and canceled out before subsequent comparisons depends on which features are shared by the options seen in the first round of comparison. Thus, changing the order in which options are seen and changing which options are seen together first has the potential to change which features are canceled out. This, in turn, will affect which features are used in later comparisons and could ultimately affect how the different options are rated. Normatively, the order in which options are seen should not affect the ratings of the options.

Two additional lines of research support the idea that changing the order in which options are seen may change preferences when feature matching is involved. First, Tversky and Gati (1978) examined carryover effects of feature matching on similarity judgments. When they presented participants with more than two choices, they found that the context in which options were presented - that is to say, the other options - affected similarity judgments. For example, when asked how similar Israel was to three other countries, including England, participants' ratings of how similar England and Israel are changed depending on what the other two comparison countries were and what features they shared with each other. Thus, Tversky and Gati concluded that similarity judgments are not independent of choice set-how features are initially clustered affects the choice context, which in turn affects similarity judgments.

The other source of research supporting the idea that shared features will remain canceled out in subsequent choices comes indirectly from work outside of the feature matching realm. Beach and his colleagues (for a review, see Beach, 1993) have conducted a number of studies demonstrating that when there are several options from which to choose, the decision can be divided into two steps: screening and actual choice. Actual choice can be based on a number of different strategies, such as satisficing and elimination by aspects but, prior to this, screening is used to weed out options from the final choice field that violate too many of the decision maker's standards.

Relevant to the idea that matched-up shared features in earlier preference decisions may not make it to later preference decisions is the finding by Beach (1993) and his colleagues that information can be "used up" during the screening process: Features that are used in the screening process to decide whether the option makes it to the final choice field may not be used in making the final choice. For example, when participants were given information with which to screen options, and then were given more information on the options that survived screening, the information used to screen contributed little to the evaluations of the options made just prior to actual choice (van Zee, Paluchowski, \& Beach, 1992). Thus, information used in screening may be used up and not available for subsequent decisions, even though it still may be retrievable from memory (van Zee, 1989, reported in Beach, 1993).

The two present studies examined the effects of feature matching and canceling on successive ratings of apartments. The first study tested the idea that if participants feature match and cancel out shared features when comparing two options, the shared features will remain canceled out, or at least exercise less influence, when subsequent comparisons are made between the original favorite of the first two options and a third option. The second study examined the robustness of the effect, introducing two possible moderating variables.

In addition, as a secondary goal, both studies attempted to uncover what is happening to the shared features when they are canceled out. If people do not use these shared features once they have canceled them out, where do they go? Given that people's evaluations do not reflect the shared features, one possibility is that the shared features are matched up, deemed use- 
less in terms of comparison, and purged from memory-a match-and-discard hypothesis.

However, Houston et al. (1989, Study 4) found evidence inconsistent with the match-and-discard hypothesis. They found that shared features were in fact more accessible in memory, which argues against the possibility that feature matching results in shared features being forgotten. Instead, the shared features may benefit because they are seen in two options rather than just one. As Houston et al. (1989, Study 4) explained, encountering shared features again in a second option serves as a retrieval cue for the shared features of the first option.

There is an alternative to the match-and-discard hypothesis that combines an explanation for why people can easily retrieve the shared features from memory but still not use them in their judgments. The shared features may be canceled out-not to be discarded, but as an organizational tactic. Because the shared features are the same, and because they do not distinguish between the two options, they are processed together, and stored by themselves, not with the unique features. This match-andfile hypothesis thus predicts that the shared features can be retrieved, if a search for them is enacted. Furthermore, if one of a shared pair is retrieved, its "match" will be, too. An analogy can be drawn from a person with a crowded desk. In order to have a space to work on the task at hand (e.g., a preference judgment), it is often necessary to file away or stack up things that are not currently being used. However, stacks and files are not like randomly discarding this material-if they are needed, they can be located and placed in the center of the desk (i.e., retrieved), where they are very visible, just as the shared features are when people are asked to recall all the features of the apartments.

\section{Study 1}

\section{Overview}

Participants were first given two apartment descriptions. For some participants, these apartment descriptions shared positive features and had unique negative features. Overall, they were relatively positive. In this condition, it was expected that participants would match up and cancel out the shared positive features and base their judgments on the remaining unique negative features. These participants were then shown a third apartment description separately, one that was less globally positive than the first two, but with entirely unique features - both positive and negative. Other participants first saw one of the more globally positive apartments paired with the less globally positive apartment. The third apartment, seen separately, was the other globally more positive apartment.

It was expected that cancellation of shared features would be more likely to occur in the first condition, when participants saw options that had matching features paired together. Because the shared (and canceled out) features of the two apartments were positive, participants should pay greater attention to the unique negative features. If the "canceling out" that occurs with feature matching persists to the next preference stage, then, relative to the condition in which cancellation was not expected to occur, ratings of the apartments with shared features should suffer (because they will be based only on the unique negatives).
The globally less positive, but entirely unique-featured apartment would look relatively better, because its ratings would be based on both positive and negative features. Thus, in a sense, feature matching and cancellation of the shared features were hypothesized to create a context in which participants would see a mediocre choice as being more positive and see positive choices as being more mediocre.

\section{Method}

Participants. One hundred twenty-nine introductory psychology students ( 88 women, 41 men) participated in exchange for course credit.

Apartment descriptions. Three apartment descriptions, each containing five positive features and three negative features, were developed in pretesting. Two of the apartments had shared positive features-that is, the five positive features were the same for these two apartments (e.g., both apartments had big windows). The negative features for these apartments were unique (e.g., one apartment had no deadbolt locks, the other had a small closet). The third apartment had all unique features. (Complete descriptions of the apartments can be found in the Appendix.) The two shared-features apartments were designed to be more positive than the all-unique-features apartment (i.e., the positive features of the two similar apartments were more positive, and the negative features were less negative, than those found in the all-unique-features apartment). In pretesting, participants were shown one of the apartment descriptions and asked to rate it. The all-unique apartment rated an average of 3.22 on a 7 -point scale (higher numbers indicate more positive ratings), as compared to shared-features Apartment 1 , rated $3.93, t(34)$ $=1.75, p=.09$, and shared-features Apartment 2 , rated $4.11, t(34)=$ $2.40, p=.03$

Procedure. On entering the lab, participants were informed that the study dealt with decision making. The experimenter asked them to imagine they had looked at several apartments that were all in the same price range and told them that they would be given notes about the different features of the apartments they had seen.

The experimenter then passed out the first apartment packet, which contained descriptions of two of the apartments, each listed on a separate page. A questionnaire about the two apartments followed the descriptions. Participants were first asked which of the two apartments was their preliminary favorite. They were then asked to rate, on 7-point scales, how positive their opinion of each apartment was $(1=$ very negative; $7=$ very positive), how likely they thought it was that they would choose to live in each of the apartments $(1=$ very unlikely; $7=$ very likely), and how much they thought they would enjoy living in each of the apartments $(1=$ not at all; $7=$ very much $) .{ }^{1}$

The experimenter collected the packets and told participants to imagine that another apartment in their price range was available and that they would be given notes to describe it, just as they had been for the other two apartments. The experimenter then distributed the second apartment packet, which contained information about the third apartment. Questions about the third apartment, identical to those asked about the first two apartments in the first packet, followed the description. ${ }^{2}$ Participants were asked once again which apartment was their favorite, this time deciding between their preliminary favorite and the third apartment. The experimenter then collected these packets.

\footnotetext{
' Participants were also asked after each question how confident they were about their answers, but asking about confidence in this way appeared to yield only an order effect, such that participants were more confident about their ratings of later apartments. These measures are not discussed further.

${ }^{2}$ Participants were also asked to rerate their preliminary favorite (but not the other apartment in the first pair). These ratings were not analyzed
} 
Feature-cancellation manipulation. Cancellation of shared features was manipulated by varying the order in which participants saw the apartments. In the feature-cancellation condition, participants first read about the two shared-features apartments together and then received the description of the all-unique-features apartment as the separate third apartment (an order expected to encourage cancellation of the first two apartments' shared features). The order of the first two apartments was counterbalanced. In the no-cancellation condition, the first pair of apartments was composed of one of the shared-features apartments along with the all-unique-features apartment. The third apartment (which participants read about separately) was the other shared-features apartment. This order was not expected to encourage feature cancellation. The order of the shared-features apartments was again counterbalanced (either in the first pair of apartments, or alone as the third apartment). Within the first pair of apartments, whether a shared-features or the all-unique apartment appeared first also was counterbalanced. ${ }^{3}$

Additional measures. After rating the apartments, participants were asked to fill out a background questionnaire that asked their sex, year in college, what their current living situation was (e.g., dorm or apartment), and what other types of places they had lived in since they came to college. The experimenter then gave participants a recall questionnaire that asked them to list as many features as they could remember for each of the apartments they had just seen. Participants were given up to $8 \mathrm{~min}$ to finish this task. When all the recall questionnaires had been collected, the experimenter passed out a questionnaire assessing participants' suspicions. Participants were then debriefed and thanked.

\section{Results}

The three dependent measures (how positive participants' opinions of the apartments were, how likely they thought they were to choose to live there, and how much they thought they would enjoy living there) were highly intercorrelated (average $r=.80$; reliability coefficients for the three different apartments ranged from .91 to .94 ). These three measures were therefore averaged to create one composite liking measure.

Feature-cancellation effects. A 3 (which apartment was rated) $\times 2$ (feature cancellation vs. no cancellation) withinbetween analysis of variance (ANOVA) on the apartment ratings revealed a main effect of apartment, $F(1,127)=4.10, p<.05$ (see means in Figure 1). Consistent with pretesting, contrasts showed that participants rated both of the shared-features apartments higher than the all-unique-features apartment: $F(1,254)$ $=8.63, p<.005$, for shared-features Apartment 1 , and $F(1$, $254)=36.67, p<.001$, for shared-features Apartment 2. Unlike in pretesting, one of the shared-features apartments (shared-

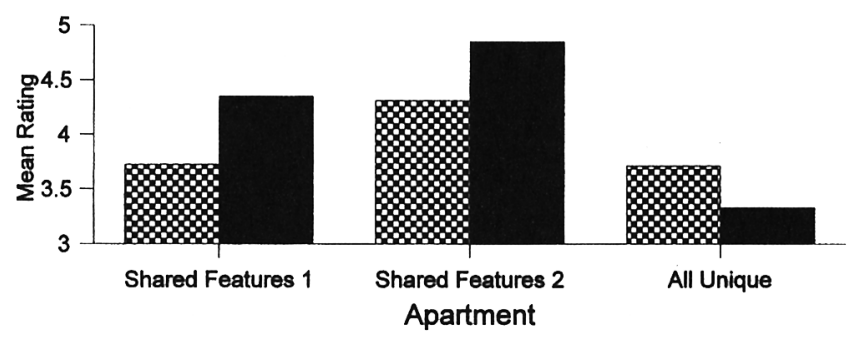

Feature Cancellation $\square$ No Cancellation

Figure 1. Apartment ratings by feature-cancellation condition, Study 1. $N=129$. features Apartment 2) was significantly preferred over the other (shared-features Apartment 1), $F(1,254)=9.72, p=.002 .^{4}$

The chief prediction concerned the interaction effect of apartment and feature-cancellation condition. As predicted, there was a significant interaction, $F(2,254)=17.73, p=.007$. To test the hypothesized pattern of results directly, a contrast was performed. First, higher weights were assigned to the two apartments with shared features when they were seen in the final position or when one was paired with the all-unique-features apartment, and lower weights were assigned to the shared-features apartments when they were paired with each other. Higher weights were assigned to the less positive, all-unique-features apartment when it appeared by itself, and lower weights were assigned when it was paired with one of the other apartments. Next, the main effect for apartment was also taken into account when determining the contrast weights so that the shared-features apartments, which were more globally positive, were more positively weighted, and the unique-features apartment, which was less globally positive, was more negatively weighted. Thus, the resulting contrast weights for the apartments in the featurecancellation condition were 0 for the two shared-features apartments and -1 for the all-unique apartment; in the no-cancellation condition, the shared-features apartments were weighted 2 , and the all-unique-features apartment was weighted -3 . This overall contrast was significant, $F(1,254)=43.47, p<.001 .^{5}$

The possibility exists, however, that this contrast was significant simply because of the strong main effect of apartment.

\footnotetext{
${ }^{3}$ An additional exploratory manipulation also was included: Some participants were explicitly told before reading about the third apartment that it was the last they would receive information on. Other participants were not told this and were led to believe that they might learn about more. There were no main effects of this manipulation, and it did not interact with the order or apartment variables; thus it is not discussed further.

${ }^{4}$ Shared-features Apartment 1 was rated nonsignificantly higher in pretesting than shared-features Apartment 2, but the difference in their ratings is more striking in the current study. It must be noted that in pretesting, participants read about only one apartment-thus, sharedfeatures Apartment 2 did not benefit from the contextual effects of being compared with two less positive rivals, which may be boosting its ratings in the present study.

${ }^{5}$ Use of the chi-square statistic revealed that the percentage of participants who picked the all-unique apartment as their favorite in the featurecancellation condition (17\%) did not differ significantly from the percentage who picked it in the no-cancellation condition $(15 \%), \chi^{2}(1, N$ $=129)<1, n s$, so although participants' ratings of the apartments were in the predicted direction, categorical choice measures-asking participants to pick their favorite apartment-were not significantly affected by feature-cancellation condition (see Dhar \& Simonson, 1992, for similar results). It is possible that despite the fact that canceling out shared features changed participants' ratings of the apartments, the initial difference between the all-unique apartment and the shared-features apartments was too great and thus not reversed in choice measures, which are generally less sensitive than judgment measures. Affecting the selection of favorites would have been a more dramatic result, but merely changing ratings still has possible repercussions, even in categorical choice situations such as elections. For example, lowered ratings of the front-runners might result in increased third-party activity and criticism of a two-party system, both of which have been suggested as aftereffects of Perot's first Presidential run.
} 
Thus, an additional contrast testing just the simple interaction of Apartment $\times$ Feature Cancellation Condition with no allowance made for the initial difference in the shared-features and allunique apartments (a more stringent test of the feature-cancellation effect) also was tested and found to be significant, $F(1$, 254 ) $=11.02, p=.001$. (Contrast weights in the feature-cancellation condition were -1 for both shared-features apartments and 2 for the all-unique apartment; weights in the no-cancellation condition were 1 for both the shared-features apartments and -2 for the all-unique apartment.)

Given that the feature-cancellation condition and the position in which an apartment appeared were necessarily confounded (e.g., one of the shared-features apartments was always seen last in the no-cancellation condition, and the all-unique apartment was always seen last in the feature-cancellation condition), serial position effects could present an alternative explanation for the difference in the ratings. Suppose for example, that people always preferred the last apartment they saw, regardless of which apartment it was. Research investigating conversational conventions (or 'Gricean'" conventions, after Grice, 1975) has demonstrated that information that comes later in an exchange is presumed to add something beyond information already exchanged, and that later information may be seen as more important than earlier information (Clark, 1989; Krosnick, Li, \& Lehman, 1990). It is conceivable that participants inferred that there was something special about the third apartment because it came last.

The order in which the options are seen is a necessary component of the hypothesized feature-cancellation effects, but a number of factors suggest that the pattern of results found in this study was not due solely to order of presentation. First, there was no linear order effect for apartment position, $F(2,254)=$ $0.44, p=.64$. More important, although the position of the allunique apartment was dictated by condition (it was always the third apartment in the feature-cancellation condition and never the third in the no-cancellation condition), the two shared-features apartments were able to appear in all three positions in the no-cancellation condition (essentially a control condition), which provided the ideal testing ground to demonstrate that there was no tendency to prefer the third apartment over the first two simply because it came last. In the four possible orders making up the no-cancellation conditions ( see orders and means in Table 1), shared-features Apartment 1 was actually preferred when it was not in the final slot $(M=3.70$ when last; $M=$ 4.95 when not last), $t(83)=-4.29, p<.01$, and sharedfeatures Apartment 2 was liked equally well whether seen last $(M=4.81)$ or not last $(M=4.89), t(83)=-0.28, p=.78$.

Aside from these results, the cover story should have reduced the likelihood that participants thought the third apartment was in any way notably different from the first two: Participants were told to imagine they were trying to choose a place to live, first with two possible options, and then again after a third option became available. Such staggered exposure is very common in real-world apartment hunts and generally does not imply any increased importance of the final option seen-only, perhaps, that the apartment seeker has just found the ad, or the apartment owner has only just put the apartment on the market. Furthermore, in a postexperimental questionnaire designed to assess questions and suspicions, only 1 participant in the sample of
129 reported any curiosity about the experimenter's motives for showing the third apartment separately.

Recall. Participants' recall scores consisted of the percentage of features they correctly recalled for an apartment. Synonyms were counted as correct (e.g. "big windows" was counted as correct for "large windows"). Any features that participants listed that were not part of the apartment's description were counted as errors.

Participants correctly recalled a higher percentage of features from the shared-features apartments ( $74 \%$ for shared-features Apartment 1 and $73 \%$ for shared-features Apartment 2) than they did of the all-unique-features apartment $(62 \%), F(2,254)$ $=18.26, p<.001 .^{6}$ Looking just at the recall for the sharedfeatures apartments, participants recalled roughly equal percentages of shared and unique features $(75 \%$ and $73 \%$, respectively), $F(1,127)=0.82, n s$, and the shared/unique-feature distinction did not interact with the feature-cancellation condition, $F(1,127)=0.57$, ns. Given that participants recalled more features from the shared-features apartments, and that they did not recall a lower percentage of shared features than unique features for these apartments (and neither of these effects was affected by feature-cancellation condition), it does not appear that participants forgot the shared features. This, like Houston et al.'s (1989) Study 4, casts considerable doubt on the canceland-discard hypothesis.

It is interesting to note, however, that the recall of features is not strictly related to the number of times a feature was seen. The shared features, despite receiving double "rehearsal" because they were seen in two descriptions, were recalled at about the same rate, not more often, as the unique features, which were seen only once. What is notable, and consistent with the match-and-file hypothesis, is that recalling a shared feature for one apartment was highly predictive of recalling it for the other, especially in the feature-cancellation condition. The average correlation between the five pairs of shared features was .82 in the feature-cancellation condition and only .27 in the no-cancellation condition $(z=4.69, p<.001)$. The average correlation between any other pair of features drawn from these two apartments was .05 in the feature-cancellation condition and .07 in the no-cancellation condition. The higher correlations between the shared features suggest that participants were pairing the shared features up, or at least forming an associative connection between them. The fact that the correlations between the shared features are even higher in the feature-cancellation condition suggests that the pairing is even stronger when the two sharedfeatures apartments are presented together. Thus, although the

\footnotetext{
"Unfortunately, several participants mistook "nosy neighbors," one of the negative characteristics of the all-unique apartment, to be "noisy neighbors." This confusion was apparent in participants' free recall, when they were asked to recall the features of the three apartments: 34 of the 129 participants listed "noisy" as one of the features of the all unique apartment in Study 1, and 35 of 191 listed it in Study 2. "Noisy" was actually one of the attributes pretested for the apartment descriptions, and it, like "nosy," was considered negative - in fact, even more so than "nosy." Thus, participants who thought that the all-unique apartment was noisy rather than nosy would judge it more harshly, making it harder for the feature-cancellation effect to occur, so it was decided not to count "noisy" as an error.
} 
Table 1

Rating of Apartments in All Six Orders, Study 1

\begin{tabular}{crrr}
\hline Condition & \multicolumn{3}{c}{ Order } \\
\hline \multirow{2}{*}{ Feature cancellation } & Shared 1: 3.86 & Shared 2: 4.25 & All unique: 3.83 \\
& Shared 2: 4.36 & Shared 1: 3.64 & All unique: 3.61 \\
No cancellation & Shared 1: 4.67 & All unique: 3.45 & Shared 2: 4.83 \\
& Shared 2: 4.85 & All unique: 2.80 & Shared 1: 3.92 \\
& All unique: 3.31 & Shared 1: 5.25 & Shared 2: 4.97 \\
& All unique: 3.81 & Shared 2: 4.95 & Shared 1: 3.44 \\
\hline
\end{tabular}

Note. Higher numbers reflect greater liking for the apartments.

double rehearsal of the shared features did not make them any more likely to be recalled than features seen only once in this study, recalling a shared feature for one apartment served as an excellent cue for recalling it for the other.

Recall errors. Of the 129 participants, 52 made at least one error in their recall (mean number of errors $=0.78, S D=1.15$ ). An ANOVA revealed a main effect of apartment on recall errors, $F(2,254)=9.88, p<.001$. A contrast, paralleling the recall data, showed that participants made more errors in recalling the features of the all-unique-features apartment $(M=0.46)$ than in recalling the features of the shared-features apartments (mean for shared-features Apartment $1=0.18$, mean for shared-features Apartment $2=0.14), F(1,254)=20.09, p<.001$.

However, unlike the recall data, there was also a significant Apartment $\times$ Feature Cancellation Condition effect, $F(2,254)$ $=3.44, p=.034$, on number of recall errors made (see means in Table 2), raising the possibility that recall errors are more sensitive to feature cancellation than is the gross number of features recalled. A post hoc contrast testing the hypothesis that participants made more errors in recalling the features of the all-unique apartment when they were in the no-cancellation condition than any other cell was significant, $F(1,254)=18.91$, $p<.001$.

\section{Discussion}

Consistent with past findings, pairing two options with shared positive features and unique negative features changed how people viewed the options. In the present study, the shared-features options were rated less positively when they were paired than if they were seen in isolation or paired with an option with which they did not share features. Furthermore, the effects of

Table 2

Mean Number of Recall Errors by Apartment and Feature Cancellation Condition, Study 1

\begin{tabular}{lcc}
\hline & \multicolumn{2}{c}{ Condition } \\
\cline { 2 - 3 } Apartment & Feature cancellation & No cancellation \\
\hline Shared 1 & $0.068^{\mathrm{a}}$ & $0.235^{\mathrm{a}}$ \\
Shared 2 & $0.204^{\mathrm{a}}$ & $0.106^{\mathrm{a}}$ \\
All unique & $0.296^{\mathrm{a}}$ & $0.541^{\mathrm{b}}$ \\
\hline
\end{tabular}

a Contrast weight $=-1 . \quad{ }^{\mathrm{b}}$ Contrast weight $=5$. feature cancellation appeared to affect subsequent decision contexts as well. When participants viewed a third, less positive option after having first had a chance to cancel out features for the two more globally positive options with shared features, their ratings of the third option were relatively more favorable than if they first saw it paired with one of the more positive options. Thus, by manipulating the context in which options are seen-specifically, manipulating which other options they are seen with, and what features they share with those other options-it is possible to change people's evaluations of the options. Furthermore, this phenomenon is not just a contrast effect based on participants liking the shared-features apartments better simply because they were paired with an inferior option in the no-cancellation condition. In this study, although one of the shared-features apartments was paired with the less globally positive all-unique apartment in the no-cancellation condition (a favorable context), the other appeared by itself in the third slot (a more neutral context).

The recall data provide some hint as to what happens to the shared features when they are matched up. It is apparent that when people cancel out shared features, they are not purging them permanently from their memories, as a match-and-discard explanation would predict. Participants in the feature-cancellation condition did just as well at recalling the shared features of the shared-features apartments as did participants in the nocancellation condition. However, they did not do any better at recalling shared versus unique features, so recall of the shared features does not appear to be simply the result of double rehearsal of these features.

The best clue to what participants are doing with the shared features comes from the correlations between recall for the two shared-features apartments. If participants recalled a shared feature for one apartment, they were highly likely to recall it for the other apartment, especially in the feature-cancellation condition. Consistent with a match-and-file explanation, if participants "pulled" the memory of a shared feature for one apartment, the memory of the other shared feature came with it. Recalling the shared feature for one apartment served as a cue to recalling it for the other apartment. However, when there was no explicit request to retrieve these features, they remained "filed away" (or at least the lower ratings of the shared-features apartments would so imply) and thus were not part of the context in which the all-unique apartment was judged.

Thus, it does not appear that feature cancellation results in people totally forgetting the shared features of the shared-fea- 
tures apartments, or in distorting their memory of the all-unique apartment in a positive direction. There was some suggestion, however, that people took the all-unique apartment more seriously in the feature-cancellation condition, because they made fewer recall errors when recalling its features, but it must be noted that they were not recalling more correct information, only including less incorrect information. Participants who viewed the all-unique apartment by itself, after matching up and canceling out the shared positive features of the shared-features apartments, may have seen the all-unique apartment as a competitive alternative and thus studied its features more carefully and made fewer errors, whereas participants who saw the all-unique apartment paired with one of the more positive shared-features apartments in the no-cancellation condition may have been more likely to make a preliminary judgment that it was inferior and thus paid less attention to it.

One possible criticism of Study 1 is that the deck was "stacked" in favor of finding a feature-cancellation effect, because participants were shown the first two apartments without being told there would be a third and because participants were asked to rate the first two apartments before seeing the third one, both of which should have encouraged them to use comparison processes that are efficient for two options. As stated earlier, canceling out shared features makes sense when we are deciding between two options - shared features do not distinguish between two options. Although Houston et al. (1991) pointed out that there are possible negative side effects of feature matching with just two options, the point of Study 1 was to show that canceling out features is particularly detrimental when subsequent comparisons are made.

Are people aware that feature matching's effectiveness is reduced in cases with more than two options? If so, explicitly telling participants that they would be seeing three options, instead of first leading them to believe that they were making a choice between only two, could make them less likely to cancel features. Alternatively, feature matching is not a strategy that we consciously choose; it is instead one that is engaged in whenever we see two options, regardless of the fact that we know we are going to see a third.

Another possible route to reducing the effects of feature matching would be to find a way to keep people from canceling out the shared features. Keeping shared features uncanceled and thus still "in the equation" should reduce feature matching effects by reducing the disproportionate influence of the unique features. For example, Sanbonmatsu, Kardes, and Gibson (1991) prevented the canceling out of shared features by explicitly instructing participants to make on-line judgments, instead of memory-based judgments (see Hastie \& Park, 1986). Presumably, because on-line participants formed global evaluations prior to comparison, they could more easily retrieve these judgments than go back to the "raw data" (the actual features).

Not allowing participants to express their preference between the first two apartments until after they see the third option could possibly serve the same function as having them make on-line judgments. People may be more likely to keep the matched-up features handy if they haven't made their ratings yet, in a manner akin to the Zeigarnik (1927/1939) effect. It is possible that making participants wait to rate the apartments would give them a reason not to discard or file away shared features. On the other hand, if feature matching is an organizational strategy in which people are not consciously choosing to engage, then making them wait to make their ratings may have no effect on whether they cancel out features: If two options with shared features are presented together, the shared features will still have been "filed" away whenever participants make their ratings. Thus, Study 2 tested whether people match and cancel shared features whenever they have a choice between two options, regardless of whether they get a chance to express a preference and regardless of what further comparisons lie ahead.

\section{Study 2}

\section{Method}

Participants. Participants were introductory psychology students who volunteered in exchange for experimental credit. Two participants' data were thrown out because their packets contained two descriptions of the same apartment. Of the 190 remaining participants, 121 were fernale, 63 were male, and 6 did not indicate their gender.

Procedure. Study 1 was replicated with the addition of two manipulations. Half of the participants were told in the first packet of two apartments that they would also later see a third apartment (knowledge condition), and the other half (no-knowledge condition) were not told in advance that they would see three apartments-they first read the two apartment descriptions and were then later presented with the third apartment (this was the procedure followed in the first study). Crossing this was a timing-of-rating manipulation. Half of the participants rated the first two apartments immediately after seeing them, as in Study 1. They rated the third apartment immediately after seeing it, and then filled out the short background questionnaire (intermittent rating condition). The other half of the participants filled out this background questionnaire in place of the first rating scale. They did not rate any of the apartments until after they had seen all three (delayed rating condition).

Participants read the same apartment descriptions as were used in Study 1 and were once again randomly assigned to either the featurecancellation or no-cancellation condition. They rated the apartments on the same liking scales.

All participants in Study 2 also performed a filler task (they were asked about how many and which experiments they had participated in during the semester) between rating the apartments and completing the free recall task. (This is because there appeared to be a possible ceiling effect for memory measures in the first study. A longer delay was inserted to increase decay of short-term memory.) One other small difference was that participants in Study 2 were run in separate cubicles, in part because they were doing more noticeably different tasks.

\section{Results}

Feature-matching effects and moderator variables. Participants' evaluations of the three apartments replicated the findings for Study 1. A 3 (which apartment was rated) $\times 2$ (feature cancellation vs. no cancellation) $\times 2$ (knowledge vs. no knowledge) $\times 2$ (intermittent rating or not) within-between ANOVA on the apartment evaluations was performed (see means in Table 3 ). Once again, there was a significant interaction between apartment and the feature-cancellation condition, $F(2,364)=$ $4.09, p=.02$ (see Figure 2). Using the same contrast weights as were used in Study 1, it was found that participants liked the shared-features apartments less in the feature-cancellation condition and the all-unique apartment more in the feature-can- 
cellation condition, $F(1,364)=36.22, p<.001 .^{7}$ The pure interaction contrast also was significant (i.e., no allowance was made for the initial ratings of the three apartments ), $F(1,364)$ $=6.73, p<.01$. As in Study $1, t$ tests conducted on the sharedfeatures apartments in the no-cancellation condition suggested that the pattern of results was not due to a simple preference for the third option seen, $t(123)=-4.24, p<.001$, for sharedfeatures Apartment 1 ( $M=3.41$ when last; $M=4.44$ when not last, meaning participants actually preferred this apartment when it did not come last), and $t(123)=-0.34, p=.731$, for sharedfeatures Apartment 2 ( $M=4.76$ when last; $M=4.83$ when not last).

In addition, as in Study 1, there was a main effect of apartment, $F(2,364)=25.84, p<.001$. The same contrasts as were performed in Study 1 showed that participants preferred sharedfeatures Apartment 1 to the all-unique apartment, $F(1,364)=$ 4.96, $p=.03$, and that participants also preferred shared-features Apartment 2 to the all-unique apartment, $F(1,364)=$ $49.55, p<.001$.

The Apartment $\times$ Feature Cancellation $\times$ Timing (intermittent or delayed rating) interaction was not significant, $F(2,364)$ $=2.08, p=.13$, but a contrast testing that the pure featurecancellation interaction was present only in the cells in which participants were not made to wait before rating the apartments was significant, $F(1,364)=10.29, p=.001$. There was also no three-way interaction among apartment, feature-cancellation condition, and knowledge condition, $F(2,364)=0.34, p=$ .714. A contrast testing that the pure feature-cancellation interaction occurred only when participants were not told they would be seeing three apartments was marginally significant, $F(1$, $364)=3.50, p=.063$.

Table 3

Apartment Evaluations by Feature Cancellation, Timing of Rating, and Knowledge About Number of Apartments

\begin{tabular}{lcc}
\hline & \multicolumn{2}{c}{ Condition } \\
\cline { 2 - 3 } Apartment & Feature cancellation & No cancellation \\
\hline & Intermittent rating \\
Doesn't know 3 & & \\
Shared 1 & $\mathbf{3 . 3 3}$ & $\mathbf{3 . 7 8}$ \\
Shared 2 & $\mathbf{3 . 7 1}$ & $\mathbf{4 . 6 6}$ \\
All unique & $\mathbf{3 . 7 6}$ & $\mathbf{3 . 2 4}$ \\
Knows 3 & & \\
Shared 1 & 3.65 & 3.97 \\
Shared 2 & 4.57 & 4.61 \\
All unique & 4.29 & 3.43 \\
\hline & & \\
& & \\
Doesn't know 3 & & 3.84 \\
Shared 1 & 3.98 & 4.98 \\
Shared 2 & 4.54 & 3.44 \\
All unique & 3.40 & 4.25 \\
Knows 3 & & 4.94 \\
Shared 1 & 3.94 & 3.46 \\
Shared 2 & 4.22 & \\
All unique & 3.20 & \\
\hline
\end{tabular}

Note. Higher numbers reflect greater liking for the apartments. Numbers in boldface type represent replication of conditions in Study 1 .

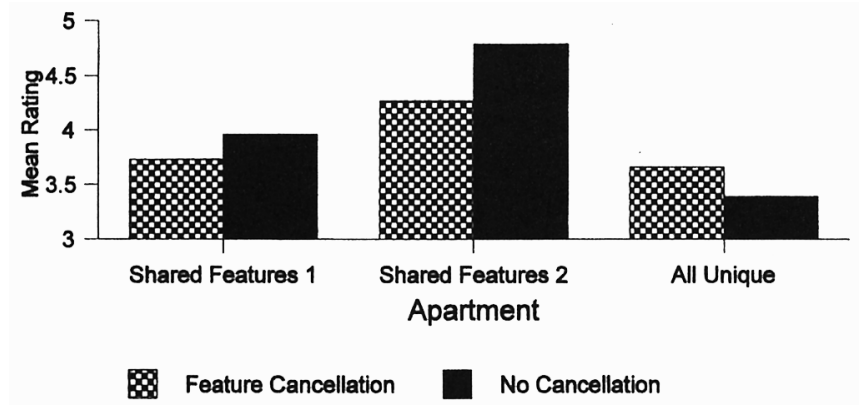

Figure 2. Apartment ratings by feature-cancellation condition, Study 2. $N=190$.

There was no main effect for either the timing of the ratings (whether participants made intermittent or delayed ratings) or for knowing that three apartments would be seen, nor was there a two-way interaction between knowledge and timing of rating. There was also no four-way interaction of apartment, featurecancellation condition, timing of rating, and knowledge of three apartments. There was an unpredicted Apartment $\times$ Timing interaction, $F(2,364)=3.04, p=.049$. An examination of the means suggests that participants liked the shared-features apartments more when they had to wait until having seen all three apartments to make their ratings, and participants liked the allunique apartment more when they made intermittent ratings of the apartments.

Recall. There was a main effect of apartment on recall results, $F(2,360)=20.57, p<.001 .^{8}$ Once again, participants recalled more of the features belonging to the shared-features apartments (69\% for shared-features Apartment 1 and $70 \%$ for shared-features Apartment 2) than they did features of the allunique apartment $(60 \%), F(1,360)=36.53, p<.001$. There were no main or interaction effects of feature-cancellation condition on number of features recalled.

In Study 2, participants recalled marginally more of the shared features $(71 \%)$ than the unique features $(66 \%)$ of the shared-features apartments, $F(1,186)=3.45, p<.065$. This pattern was also not affected by feature-cancellation condition. Thus, once again, there was no support for the match-and-discard hypothesis, whereby people match up features and purge them from memory. Most striking, however, was the support for the match-and-file hypothesis, by means of a replication of the Study 1 finding that recalling one of the shared features for one apartment was highly predictive of recalling it for the other in the feature-cancellation condition. The average correlation between the five pairs of shared features was .67 in the featurecancellation condition and only .30 in the no-cancellation condition $(z=3.27, p=.001)$. The average correlation between any

\footnotetext{
${ }^{7}$ As in Study 1, there was no reliable difference between the percentage of participants in the feature-cancellation condition $(20 \%)$ and the no-cancellation condition $(16 \%)$ who picked the all-unique apartment as their favorite, $x^{2}(1, N=190)<1$, ns. The additional independent variables also did not affect participants' categorical choices.

${ }^{8}$ Slightly different $n$ s reflect participants who did not complete recall measures.
} 
other pair of features drawn from these two apartments was .12 in both the feature-cancellation condition and no-cancellation condition. The possible moderator variables - timing of ratings and knowledge that three options would be seen-had no significant effect on the correlations.

Recall errors. As in Study 1, there was a main effect of apartment on recall errors, $F(2,360)=5.34, p=.005$. A contrast showed that participants made many more errors in their recall of the features belonging to the all-unique apartment, $F(1,186)=7.82, p=.006$. This effect was strongest in the no-cancellation condition (see means in Table 4), replicating the significant Apartment $\times$ Feature Cancellation Condition interaction found in Study 1, $F(2,360)=7.20, p=.001$.

\section{Discussion}

Study 2 closely replicated Study 1 . When two options with shared positive features and unique negative features were paired, they were rated lower than when they were seen either alone or paired with an option with nonshared features. The effect of pairing these two options also carries over to subsequent comparisons: A less positive option with all unique features benefited from being seen after the paired shared-features options. In addition to the evaluative ratings of the apartments, Study 2 replicated Study 1's results for recall and recall errors.

Furthermore, the results from Study 2 suggest that the conditions chosen for Study 1 are not the only ones under which the feature-cancellation effect can be obtained. Attempts to derail this robust effect in Study 2 were weak at best. As can be seen in Table 3, the pattern of means essentially followed the featurecancellation effect in all cells (with the exception of the ratings of the all-unique apartment when participants were not allowed to make intermittent ratings ), and the feature-cancellation effect was significant when the data were collapsed across the knowledge and timing-of-rating variables. Furthermore, the correlations for memory of shared features were unaffected when participants had to wait to make their ratings.

The significant contrast testing that the cancellation of shared features occurred only in the cells in which participants made intermittent ratings suggests that this manipulation could strengthen the effect. To use the crowded-desk analogy again, this could occur because the "file" of matched features has more of a chance to become buried among other things on the desktop when participants make intermittent ratings. Consistent with this hypothesis, the minimal dampening effect of not allowing intermittent ratings in the feature-cancellation condi-

Table 4

Mean Number of Recall Errors by Apartment and Feature Cancellation Condition, Study 2

\begin{tabular}{lcc}
\hline & \multicolumn{2}{c}{ Condition } \\
\cline { 2 - 3 } Apartment & Feature cancellation & No cancellation \\
\hline Total errors & & \\
Shared 1 & 0.246 & 0.285 \\
Shared 2 & 0.338 & 0.236 \\
All unique & 0.262 & 0.691 \\
\hline
\end{tabular}

tion appears to have its strongest effect on subsequent choices (i.e., the ratings of the all-unique apartment). Even with a delay, participants were matching up and canceling out the shared features of the shared-features apartments, as evidenced by the fact that the ratings of these two apartments still suffered in the feature-cancellation condition relative to the no-cancellation condition and the fact that the correlations between memory for the shared features was not affected by the timing of the ratings. However, delaying the ratings may have kept the shared features closer at hand and thus reduced the contextual effect of cancellation on the ratings of the all-unique apartment when it came last. The marginal effect of the delay also offers further evidence against a Gricean preference for the third option (as discussed in Study 1), as it seems unlikely that the timing of the judgments should change the participants' assumptions about what the experimenter intended to communicate by providing the description of the third apartment separately.

An alternative method of examining this hypothesis would be to make participants wait to rate the apartments but to insert a distraction task before they got a chance to do so, to see if that, too, made the shared features less of a factor in judgments. The more readily visible the shared features are as a part of the context against which the all-unique apartment is judged, the less of a cancellation effect there should be. However, it appears that the mere act of comparison automatically sets a process into motion that reduces the salience of these shared features.

\section{General Discussion}

Feature matching can be seen as a strategy with qualities that are analogous to Beach's (1993) investigations of choice screening: As with screening, when people feature match, some of the initial information is lost when people make their "first round" of comparison, even though that information might be valuable to have for later comparisons. In addition to demonstrating the effects of feature matching on successive decisions, the present studies shed light on the mediating mechanism. Given the recall data, people in the feature-cancellation condition apparently do not match up the information to throw it out, as a match-and-discard hypothesis would predict. As in the screening studies, the information used in earlier comparisons is not forgotten (in one of the studies Beach discussed, participants were reminded of the screened information but still did not use it) but, judging from people's ratings, it does not factor as heavily as the unique features into people's evaluations of the apartments.

Instead, the matching and cancellation appears to be more of an organizational match-and-file strategy, as a means to sort through many pieces of incoming information. If this is indeed the model for feature matching, then we should expect cognitive correlates, such as reaction time differences. For example, it should take longer to retrieve a shared feature for the first option whose features are to be recalled than for the second option, because for the first option the feature must be retrieved from the "filing cabinet," whereas for the second it will already be "on the desk."

Another possible way of testing the hypothesis that people are clearing the shared features from their cognitive workspace would be to give them a task that required them to use the shared 
features in addition to having to make preference judgments. As mentioned earlier, Tversky's (1977) original studies discussed feature matching as a strategy for judging similarity. In the case of similarity judgments, the shared features were not canceled out - the number of shared features was the first estimate of similarity. Thus, if people knew they had to make similarity judgments in addition to preference judgments, they might be less likely to cancel out or file away the shared features, because the shared features are a necessary component for making similarity judgments.

\section{Do People Know When They Feature Match?}

As an exploratory exercise, a subset of 110 of the participants in Study 2 were asked at the end of the study (during the debriefing but before feature matching was explained to them) how they made their ratings and whether they were affected differently by the shared and unshared features. Despite the questionable validity of asking people to verbalize their cognitive processes (Nisbett \& Wilson, 1977; Wilson, 1994), especially after the fact (Ericsson \& Simon, 1980; Payne, 1994), it is still interesting to examine the protocols of participants who said they used strategies. Approximately $40 \%$ of the participants asked about their strategies described something that had to do with feature matching; some of them gave textbook descriptions of matching and canceling the shared features (e.g., a participant in the feature-cancellation condition said "The shared features were easy to put aside because I could then focus on differences between the apartments"). However, there were participants in both the feature-cancellation condition and those in the nocancellation condition who claimed to have done something along the lines of matching up and canceling shared features, looking just at unshared features, or both (e.g., from a participant in the no-cancellation condition: "I think that the shared features allowed me to clump those apartments together by shared characteristics. They forced me to focus even more on the bad of each complex" ). If anything, cancellation strategies were self-reported more in the no-cancellation condition.

Although the self-reports were meant to be exploratory only, they are suggestive evidence that canceling out shared features is dangerous to the quality of preference judgments only in cases where there is a separation between the initial matching and subsequent choices - when there is time for the shared-features "file" to get put away. Participants in both conditions said that they matched and canceled, but only participants who had a separation between the shared-features apartments and the allunique apartment (either because the shared-features apartments were both paired and rated together, as in the intermittent rating condition, or just paired together, as in the delayed rating condition ) strayed from normative ratings.

\section{Is This "Shortcut" All That Short?}

Suggesting that people are going to the effort of both matching and filing shared features raises the question of whether feature matching is really a shortcut or heuristic at all. Presumably, both matching and filing require some cognitive capacity, possibly even more than not using any strategy. At least for the first round of comparison in the feature-matching paradigm described in these studies, the heuristic people are using is not so much a shortcut that allows them to skip over parts of the decision process but is more a strategy that makes choices seem clearer. People are neither ignoring options, as in satisficing, or even ignoring features, as in elimination by aspects. They instead look at all the information, but they structure it in such a way that makes it easier to compare.

This interpretation would be consistent with other studies that suggest that people are motivated to do more than just be "cognitive misers" (Fiske \& Taylor, 1991) in order to make decisions easier. For example, Simonson and Tversky (1992) theorized that trade-off contrast and extremeness aversion, two other decision-making context effects, may be "driven primarily by an attempt to achieve better resolution and identify the best choice, not merely by the tendency to simplify the task' (p. 293; see also Montgomery, 1989, and Tversky \& Shafir, 1992). Thus, it is possible that there is a curvilinear relationship between feature matching and the cognitive load imposed by judgment: Matching and canceling appears only when making the judgment is complex enough that people wish to simplify it but not so complex that they do not have the cognitive capacity to perform the matching.

Related to this question, another area of future investigation would be to examine how much cognitive effort we are willing to exert to feature match. For example, suppose the shared features of the two shared-features apartments were not identically worded. If "spacious" became "lots of space" in one apartment and "generous floor plan" in the other, would people still make the match? Would they be more likely to do so when they had more features to look at (and thus motivated to "file" some away, in order to deal with a more reasonable "stack")?

Besides varying the level of effort required to feature match, there are other limiting circumstances under which people would not be expected to cancel out shared positives and subsequently would make the mistake of boosting their ratings of later inferior options. Feature matching would not be expected to occur (let alone have adverse effects on decision quality) when a person had a distinct preference for one of two options, just as other contextual influences have been shown to be less influential with well-established preferences (see Huber, Payne, \& Puto, 1982; Simonson \& Tversky, 1992). A lifelong, die-hard Democrat probably would not have been swayed to vote for Perot: To a Democrat, Clinton would still be a Democrat, even if all of his other positive features overlapped with Bush's.

Various other motivations also could influence the degree to which people cancel shared features. Need for closure, for example, might increase people's likelihood to cancel features (Kruglanski \& Weber, 1996), whereas accountability might decrease it (Simonson \& Nye, 1992). Other aspects of the decision context could also play a role in people's likelihood to cancel features. For example, presenting one of the shared-features options earlier than the other, so it is viewed as a "pioneer" (see Kardes \& Gurumurthy, 1992), or asking participants to judge the similarity of the two shared-features options (which should make the shared features more salient) might reduce feature-cancellation effects.

In conclusion, the research in the present studies is consistent with a growing body of work that casts doubt on the idea that human judgments are absolute. It appears we cannot just read 
how much we like something off a "preference thermometer;" instead preferences "are . . . constructed-not merely revealed — during their elicitation"' (Shafir, 1993, p. 546; see also Shafir, Simonson, \& Tversky, 1993). This construction is affected by the context in which our judgments are made. Changing things such as the order in which options are seen and the number of features they have in common, as well as those they possess uniquely, can cause our ratings to stray from normative models, providing yet another example of efficient yet imperfect human judgment (see Payne, Bettman, \& Johnson, 1988). The present studies even suggest that it is our own structuring of the decision context, in an attempt to make the decision easier or clearer, that can "mess us up." The structure may outlive its usefulness; highlighting certain relative differences may obscure absolute differences. Perhaps, however, in many of our decisions, the good closure we feel when a decision is clear to us is more psychologically important in the long run than always picking the normative choice.

\section{References}

Beach, L. R. ( 1993). Broadening the definition of decision making: The role of prechoice screening of options. Psychological Science, 4, 215 220.

Clark, H. H. (1989). Language use and language users. In G. Lindzey \& E. Aronson (Eds.), The handbook of social psychology (Vol. 2, pp. 179-231). New York: Random House.

Dhar, R., \& Simonson, I. (1992). The effect of the focus of comparison on consumer preferences. Journal of Marketing Research, 29, 430-440.

Ericsson, K. A., \& Simon, H. A. (1980). Verbal reports as data. Psychological Review, 87, 215-251.

Fiske, S. T., \& Taylor, S. E. (1991). Social cognition. New York: McGraw-Hill.

Grice, H. P. (1975), Logic and conversation. In P. Cole \& J. L. Morgan (Eds.), Syntax and semantics (Vol. III): Speech acts. New York: Academic Press.

Hastie, R., \& Park, B. (1986). The relationship between memory and judgment depends on whether the judgment task is memory-based or on-line. Psychological Review, 93, 258-268.

Houston, D. A., \& Sherman, S. J. (1995). Cancellation and focus: The role of shared and unique features in the choice process. Journal of Experimental Social Psychology, 31, 357-378.

Houston, D. A., Sherman, S. J., \& Baker, S. M. (1989). The influence of unique features and direction of comparison on preferences. Journal of Experimental Social Psychology, 25, 121-141.

Houston, D. A., Sherman, S. J., \& Baker, S. M. (1991). Feature matching, unique features and the dynamics of the choice process: Predecision conflict and postdecision satisfaction. Joumal of Experimental Social Psychology, 27, 411-430.

Huber, J., Payne, J. W., \& Puto, C. (1982). Adding asymmetrically dominated alternatives: Violations of regularity and the similarity hypothesis. Journal of Consumer Research, 9, 90-98.

Kahneman, D., \& Tversky, A. (1984). Choices, values and frames. American Psychologist, 39, 341-350.

Kardes, F. R., \& Gurumurthy, K. (1992). Order-of-entry effects on consumer memory and judgment: An information integration perspective. Journal of Marketing Research, 29, 343-357.
Kardes, F. R., \& Sanbonmatsu, D. M. ( 1993 ). Direction of comparison, expected feature correlation, and the set-size effect in preference judgment. Journal of Consumer Psychology, 2, 39-54.

Krosnick, J, A., Li, F., \& Lehman, D. R. (1990). Conversational conventions, order of information acquisition, and the effect of base rates and individuating information on social judgments. Journal of Personality and Social Psychology, 59, 1140-1152.

Kruglanski, A. W., \& Weber, D. M. (1996). Motivated closing of the mind: "Seizing"' and "freezing." Psychological Review, 103, 263283.

Luce, R. D. (1959). Individual choice behavior. New York: Wiley.

Montgomery, H. (1989). From cognition to action: The search for dominance in decision making. In $\mathrm{H}$. Montgomery \& O. Svenson (Eds.) Process and structure in human decision making (pp. 23-49). New York: Wiley.

Nisbett, R. E., \& Wilson, T. D. (1977). Telling more than we can know: Verbal reports on mental processes. Psychological Review, 84, 231-259.

Payne, J. W. (1994). Thinking aloud: Insights into information processing. Psychological Science, 5, 241, 245-248.

Payne, J. W., Bettman, J. R., \& Johnson, E. J. ( 1988). Adaptive strategy selection in decision making. Journal of Experimental Psychology: Learning, Memory and Cognition, 14, 534-552.

Restle, F. (1961). Psychology of judgment and choice: A theoretical essay. New York: Wiley.

Rumelhart, D. L., \& Greeno, J. G. (1971). Similarity between stimuli: An experimental test of the Luce and Restle choice models. Journal of Mathematical Psychology, 8, 370-381.

Sanbonmatsu, D. M., Kardes, F. R., \& Gibson, B. D. (1991). The role of attribute knowledge and overall evaluations in comparative judgment. Organizational Behavior and Human Decisions Processes, 48, 131-146.

Shafir, E. (1993). Choosing versus rejecting: Why some options are both better and worse than others. Memory \& Cognition, 21, 546-556.

Shafir, E., Simonson, I., \& Tversky, A. (1993). Reason-based choice. Cognition, 49, 11-36.

Simonson, I., \& Nye, P. (1992). The effect of accountability on susceptibility to decision errors. Organizational Behavior and Human Decision Processes, 51, 416-446.

Simonson, I., \& Tversky, A. (1992). Choice in context: Tradeoff contrast and extremeness aversion. Journal of Marketing Research, 29, 281295.

Skowronski, J. J., \& Carlston, D. E. (1989). Negativity and extremity biases in impression formation: A review of explanations. Psychological Bulletin. 105, 131-142.

Tversky, A. (1972). Elimination by aspects: A theory of choice. Psychological Review; 79, 281-299.

Tversky, A. (1977). Features of similarity. Psychological Review, 84, 327-352.

Tversky, A., \& Gati, I. (1978). Studies of similarity. In E. Rosch \& B. Lloyd (Eds.), Cognition and categorization (pp. 81-98). Hillsdale, NJ: Erlbaum.

Tversky, A., \& Shafir, E. (1992). Choice under conflict: The dynamics of deferred decision. Psychological Science, 3, 358-361.

van Zee, E. H., Paluchowski, T. F., \& Beach, L. R. (1992). The effects of screening and task partitioning upon evaluations of decision options. Journal of Behavioral Decision-Making, 5, 1-23.

Wilson, T. D. (1994). The proper protocol: Validity and completeness of verbal reports. Psychological Science, 5, 249-252.

Zeigarnik, B. (1927/1939). On finished and unfinished tasks. In W. D. Ellis (Ed.), A sourcebook of gestalt psychology (pp. 300-314). New York: Harcourt, Brace. 
Appendix

Apartment Descriptions

\begin{tabular}{|c|c|c|}
\hline $\begin{array}{l}\text { Apartment 1: Shared } \\
\text { positive features/unique } \\
\text { negative features }\end{array}$ & $\begin{array}{l}\text { Apartment 2: Shared } \\
\text { positive features/unique } \\
\text { negative features }\end{array}$ & $\begin{array}{l}\text { Apartment 3: } \\
\text { All unique features/ } \\
\text { less globally positive }\end{array}$ \\
\hline Pool & Big windows & Wall-to-wall carpet \\
\hline Great deck & No deadbolt locks & Limited parking \\
\hline Ants & Pool & Renovated old building \\
\hline Spacious & Laundry facilities on premises & Nosy neighbors \\
\hline Torn screens & No cable & Lots of phone jacks \\
\hline Big windows & Great deck & Damp in the summer \\
\hline Small closet & Drafty in winter & Separate dining room \\
\hline Laundry facilities on premises & Spacious & Walking distance to grocery store \\
\hline
\end{tabular}

Received September 15, 1995

Revision received August 30, 1996

Accepted September 10, 1996

\section{American Psychological Association SUBSCRIPTION ClaIMS INFORMATION}

Today's Date:

We provide this form to assist members, institutions, and nonmember individuals with any subscription problems. With the appropriate information we can begin a resolution. If you use the services of an agent, please do NOT duplicate claims through them and directly to us. PLEASE PRINT CLEARLY AND IN INK IF POSSIBLE.

\begin{tabular}{ll}
\hline PRINT FULL NAME OR KEY NAME OF INSTTIUTION & \\
\hline ADDRESS & \\
\hline STATE/COUNIRY & ZIP \\
\hline
\end{tabular}

YOUR NAME AND PHONE NUMBER

TITLE

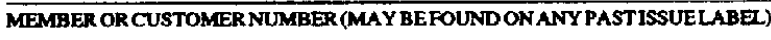

DATE YOUR ORDER WAS MAILED (OR PHONED)

\section{PREPAID _C CHECK CHARGE}

CHECKMCARD CLEARED DATE

(If possible, send a copy, front and back, of your cancelled ebeck to belp us in our research of your cleim.)

VOLUME OR YEAR

Thank you. Once a claim is received and resolved, delivery of replacement issues routinely takes $4-6$ weeks.

(TO BE FILLDD OUT BY APA STAFI)

DATE RECEIVED;

DATE OF ACTION:

ACTION TAKEN:

INV. NO. \& DATE:

STAFF NAME:

LABEL NO. \& DATE:

Send this form to APA Subscription Claims, 750 First Street, NE, Washington, DC 20002-4242

PLEASE DO NOT REMOVE. A PHOTOCOPY MAY BE USED. 